\title{
Práticas ambientalmente sustentáveis em unidades de alimentação e nutrição hospitalares
}

\section{Environmentally sustainable practices in hospital foodservices}

\author{
Katrina Skolove Silva1 ${ }^{1}$ Angélica Cotta Lobo Leite Carneiro', \\ Leandro de Morais Cardoso ${ }^{1 *}$ (i) \\ ${ }^{1}$ Universidade Federal de Juiz de Fora (UFJF), Departamento de Nutrição, Governador Valadares/MG - Brasil \\ ${ }^{*}$ Corresponding Author: Leandro de Morais Cardoso, Universidade Federal de Juiz de Fora (UFJF), \\ Departamento de Nutrição, Campus Governador Valadares, Rua Manoel Byrro, 241, Vila Bretas, CEP: 35032-620 \\ Governador Valadares/MG - Brasil, e-mail: leandro.cardoso@ufjf.br
}

Cite as: Silva, K. S., Carneiro, A. C. L. L., \& Cardoso, L. M. (2022). Environmentally sustainable practices in hospital foodservices. Brazilian Journal of Food Technology, 25, e2020091. https://doi.org/10.1590/19816723.09120

\begin{abstract}
Resumo
Este estudo analisou as ações realizadas por Unidades de Alimentação e Nutrição - UAN hospitalares para a promoção da sustentabilidade ambiental. Participaram do estudo quatro das seis UANs de hospitais do município de Governador Valadares-MG. A coleta de dados foi realizada utilizando questionário online composto por 73 questões estruturadas sobre práticas de sustentabilidade ambiental na UAN. As ações estruturais menos prevalentes nos estabelecimentos foram as relacionadas a energia e iluminação (25\%), e ventilação (38\%). Aproximadamente, $93 \%$ das ações durante o planejamento de cardápio eram realizadas pelos estabelecimentos, porém $25 \%$ dos estabelecimentos não possuíam fichas técnicas de preparação. Apenas $35 \%$ das ações eram realizadas pelas unidades durante a aquisição e o armazenamento de insumos, sendo que nenhuma utilizava alimentos da agricultura familiar ou alimentos orgânicos. Durante o preparo, a distribuição e o consumo das refeições, $75,0 \%$ das ações realizadas se relacionaram ao aproveitamento integral dos alimentos. O controle de temperatura durante a distribuição de refeições foi a ação menos prevalente (50\%). As unidades realizavam, em média, 93,8\% das ações relacionadas aos procedimentos de higienização. Em relação a geração, coleta, armazenamento e destinação de resíduos, em média, 50\% das ações eram adotadas nas UANs. Conclui-se que as UANs hospitalares de Governador Valadares-MG adotam medidas para a promoção da sustentabilidade ambiental, porém estas devem ser intensificadas, especialmente as relacionadas a capacitação da equipe, estrutura física, aquisição e recepção de insumos, e gestão de resíduos.
\end{abstract}

Palavras-chave: Meio ambiente; Sustentabilidade; Sustentabilidade ambiental; Práticas sustentáveis; Nutrição; Serviços de alimentação.

\section{Abstract}

This study analyzed the actions taken by hospital Foodservices to promote environmental sustainability. Four of the six Foodservices of hospitals in the municipality of Governador Valadares-MG/Brazil participated in the study. Data collection was performed online by using a questionnaire composed of seventy-three dichotomous and structured 
questions, thus being grouped into an identification and characterization of axis regarding the establishment and also into six axes on environmental sustainability practices in FS. The least prevalent physical structural actions in establishments were those related to energy and lighting (25\%) and ventilation (38\%). Approximately $93 \%$ of the actions during menu planning were carried out by the establishments, however, $25 \%$ of the establishments did not have technical preparation sheets. Only $29.1 \%$ of the actions during the acquisition and receipt of inputs were carried out by the units, however, none of which facilitated the use of organic food or purchased food from family farm. During preparation, distribution and consumption of meals, adherence to actions to promote environmental sustainability was $75.0 \%$, with full use of food and temperature control during meal distribution being the least prevalent (50.0\%). Regarding the generation, collection, storage and disposal of waste, it could be noted that, on average, $52.2 \%$ of the actions were adopted by the FS. This study concluded that the hospital Foodservices of Governador Valadares hospitals (MG/Brazil) adopted measures aimed at promoting environmental sustainability, however, these measures must be intensified, especially those related to staff training, physical structure, acquisition and reception of inputs as well as waste management.

Keywords: Environment; Sustainability; Environmental sustainability; Sustainable practices; Nutrition; Food services.

\section{Introdução}

Os danos ambientais que ocorrem nos níveis individual, institucional e social são um dos maiores problemas do mundo e resultam, principalmente, de um comportamento humano inconsciente (Yucedag et al., 2018). Diante deste contexto, articulações para o desenvolvimento ambiental sustentável de comunidades, indústrias e governos têm sido realizadas visando equilibrar as necessidades humanas com um ambiente finito, através da aplicação de padrões e de técnicas que racionalizem o uso de recursos naturais e os preservem para as próximas gerações (Moskwa et al., 2015).

As práticas existentes de produção, distribuição e consumo de alimentos são provavelmente insustentáveis e resultam em elevado impacto ambiental (Hatjiathanassiadou et al., 2019). Ao longo desta cadeia de produção de alimentos, as Unidades de Alimentação e Nutrição, sejam elas comerciais ou institucionais, com ou sem fins lucrativos, realizam atividades que poderão impactar o meio ambiente (Llach et al., 2013). Entre as UANs de diferentes segmentos, aqueles presentes em instituições hospitalares desempenham um papel importante no sistema alimentar devido aos recursos financeiros que movimentam, ao uso de recursos naturais e à geração de resíduos (Payne-Palacio \& Theis, 2015). Destaca-se que hospitais têm a responsabilidade de apoiar a saúde humana e, dada a ligação entre a saúde humana e meio ambiente, os hospitais e seus setores devem considerar seus impactos ambientais.

As UANs hospitalares produzem refeições destinadas a pacientes, bem como podem oferecer alimentação gratuita ou paga aos funcionários, acompanhantes e visitantes (Payne-Palacio \& Theis, 2015). Estas unidades podem afetar negativamente o ambiente em todas as fases da cadeia de abastecimento alimentar (produção/aquisição, distribuição, preparação, consumo e gestão / eliminação de resíduos), uma vez que a produção de refeições destinada a uma elevada quantidade de clientes requer vastos recursos (por exemplo, energia, água e terra) e pode gerar emissões e resíduos em cada etapa do processo de produção. Por exemplo, existem implicações ambientais decorrentes do tipo de preparações presentes no cardápio, da origem das matérias-primas, da logística de transporte, do uso de materiais não descartáveis e da gestão de alimentos e resíduos (Carino et al., 2020).

Em revisão sistemática realizada por Carino et al. (2020), constatou-se que o conhecimento sobre as ações adotadas em UANs hospitalares tem se concentrado amplamente nas fases de consumo e aquisição, especificamente no desperdício de alimentos e na compra local de alimentos. Além disso, os autores observaram a incipiência da literatura sobre as estratégias ambientalmente sustentáveis adotadas nas etapas de distribuição, preparação e gestão de resíduos (Carino et al., 2020). A maioria dos estudos em estabelecimentos brasileiros publicados até o momento se restringe a avaliar restaurantes comerciais e UANs industriais, e enfoca os mesmos temas observados por Carino et al. (2020) (Araújo \& Carvalho, 2015; Silva, 
2017; Viana \& Ferreira, 2017; Zotesso et al., 2016). Desta forma, existe uma lacuna de conhecimento sobre as práticas visando à redução de impactos ambientais adotadas em UANs brasileiras em geral, incluindo as hospitalares, durante o planejamento de cardápios, a aquisição e o armazenamento de matérias-primas, o preparo, a distribuição e o consumo de refeições, bem como o planejamento e a gestão de estruturas físicas.

Considerando que a busca por estratégias que reduzam os impactos ambientais negativos no setor de alimentação coletiva é fundamental para oferecer refeições não só saudáveis, mas também sustentáveis (Hatjiathanassiadou et al., 2019), e diante da necessidade de se diagnosticar o perfil ambiental dos serviços de alimentação (González-García et al., 2020), este estudo avaliou as práticas de sustentabilidade ambiental em Unidades de Alimentação e Nutrição hospitalares em Governador Valadares, Minas Gerais.

\section{Materiais e métodos}

Tratou-se de um estudo descritivo, transversal e qualitativo, conduzido entre os meses de junho e agosto de 2018 .

\subsection{Participantes}

Participaram do estudo quatro Unidades de Alimentação e Nutrição de hospitais, públicos e privados, localizados no município de Governador Valadares-MG. O convite aos nutricionistas, responsáveis técnicos dos estabelecimentos, foi realizado por e-mail, sendo incluídos somente aqueles que assinaram o Termo de Consentimento Livre e Esclarecido (TCLE).

\subsection{Coleta de dados}

Para a coleta de dados, a lista de verificação de boas práticas ambientais em serviços de alimentação elaborada por Colares et al. (2018) foi utilizada, com a inclusão de questões complementares para a caraterização das UANs. A lista foi composta por 73 questões estruturadas, dicotômicas e de preenchimento obrigatório. As questões foram agrupadas em um eixo de identificação e caracterização da UAN, e seis eixos sobre práticas de sustentabilidade ambiental em UAN: 1) estrutura física (água, esgoto, equipamentos, iluminação, ventilação, energia e edificação); 2) planejamento de cardápios; 3) aquisição e armazenamento de insumos; 4) preparação de refeições; 5) distribuição e consumo de refeições; 6) gestão de resíduos (coleta, armazenamento e destinação).

A lista de verificação foi disponibilizada ao nutricionista via Google Forms para preenchimento em um prazo de até uma semana.

\subsection{Análise de dados}

Os dados foram tabulados automaticamente pelo Google Forms utilizando o software Microsoft Office Excel®, versão 2016. Os resultados foram apresentados pela frequência absoluta e relativa de cada questão avaliada e da média geral de todas as questões de cada eixo de avaliação.

\subsection{Aspectos éticos}

A pesquisa foi aprovada pelo Comitê de Ética em Pesquisa com Seres Humanos da Universidade Federal de Juiz de Fora (CAAE $n^{\circ}$ 77985117.1.0000.5147) e cumpriu as diretrizes e normas que regem as pesquisas envolvendo seres humanos (Resolução nº 196/96). 


\section{Resultados e discussão}

\subsection{Caracterização das UANs}

Participaram do estudo, aproximadamente, 66\% das UANs hospitalares do município de Governador Valadares $(\mathrm{n}=4)$, as quais pertenciam majoritariamente a hospitais privados $(75 \%, \mathrm{n}=3)$. As UANs participantes produziam, em média, 1.320 refeições de padrão popular e possuíam entre 18 e 30 funcionários. Todas as UANs possuíam até dois nutricionistas e 50\% tinham o mesmo nutricionista responsável técnico havia mais de seis anos.

Verificou-se que $50 \%$ das UANs possuíam nutricionistas capacitados quanto à sustentabilidade ambiental ou promoviam ações educativas para conscientizar os clientes sobre uso adequado de energia e de água. Em $75 \%$ dos estabelecimentos, os funcionários eram capacitados visando ao manejo adequado de resíduos e ao uso racional de água e de energia. A promoção da sustentabilidade em UANs constitui uma construção coletiva que perpassa pela capacitação de todos os envolvidos no processo de produção e consumo de refeições. Neste processo, os profissionais de nutrição devem atuar como influenciadores do desenvolvimento sustentável na UAN fazendo com que a gestão de recursos humanos assuma o seu papel estratégico para a promoção da sustentabilidade e que os colaboradores e clientes da unidade sejam capacitados regularmente (Süßbauer et al., 2019).

\subsection{Estrutura física}

Em média, 41,2\% das ações relacionadas à estrutura física eram realizadas pelas UANs avaliadas (Tabela 1). A adoção de medidas estruturais para a promoção da sustentabilidade pode contribuir sobremaneira para reduzir os impactos deletérios sobre os recursos naturais. Estima-se, por exemplo, que a construção de uma edificação sustentável pode reduzir o consumo de energia em $30 \%$, a emissão de carbono em $35 \%$ e o uso de água de $30 \%$ a $50 \%$ (Block, 2019).

Quanto às medidas estruturais relacionadas, o uso racional de água e a identificação e a correção de vazamentos na rede foram as ações mais prevalentes nos estabelecimentos avaliados. Em contrapartida, em apenas uma unidade a água da chuva era coletada e reutilizada. As iniciativas para a reutilização e o reuso de água constituem importantes práticas de racionalização e de conservação de recursos hídricos, atuando de forma direta na disponibilidade deste recurso natural e na preservação e conservação do meio ambiente (Sgroi et al., 2018). Além disso, ações para reduzir o risco de contaminação da água com resíduos gerados pela UAN também devem ser adotadas de maneira eficaz.

A maioria das unidades buscava adquirir equipamentos com maior eficiência energética e possuía procedimentos operacionais padronizados (POPs) para a manutenção dos equipamentos. Porém, apenas $50 \%$ dos estabelecimentos realizavam a calibração dos termômetros e a manutenção em sistemas de climatização, e registravam a ocorrência da manutenção em equipamentos em geral. A aquisição de equipamentos modernos e energeticamente eficientes aliada a manutenções preventivas periódicas e corretivas imediatas constituem importantes estratégias para o uso racional de energia. Destaca-se ainda que as ações em equipamentos que visam garantir a temperatura de alimentos durante o processo de produção contribuem diretamente para a qualidade higienicossanitária da refeição e, desta forma, para a promoção das dimensões ambiental, social e econômica da sustentabilidade. 
Tabela 1. Práticas de sustentabilidade ambiental relacionadas à estrutura física adotadas em Unidades de Alimentação e Nutrição de hospitais em Governador Valadares-MG, agosto de 2018.

\begin{tabular}{|c|c|c|}
\hline Consumo de Água & $\%$ & $\mathbf{n}$ \\
\hline Identificação e correção de vazamento na rede de água e nos equipamentos & 100 & 4 \\
\hline Hidrômetros individuais de água (por área de processamento de alimentos) & 50 & 2 \\
\hline Torneiras com arejadores & 50 & 2 \\
\hline Duchas com redutor de vazão & 25 & 1 \\
\hline Há mictório com válvula automática & 0 & 0 \\
\hline Bacia sanitária com caixa acoplada e acionador com dois botões & 25 & 1 \\
\hline Reaproveitamento e reuso de água & 25 & 1 \\
\hline Coleta e reutilização de água de chuva & 25 & 1 \\
\hline Uso da própria água da caixa d'água no momento de sua limpeza & 0 & 0 \\
\hline Tratamento e reuso de água cinza & 0 & 0 \\
\hline Esgoto & $\%$ & $\mathbf{n}$ \\
\hline Caixas de gordura em adequada localização e adequado estado de conservação e de funcionamento & 100 & 4 \\
\hline Fossas, esgotos conectados à rede pública & 75 & 3 \\
\hline Equipamentos & $\%$ & $\mathbf{n}$ \\
\hline Aquisição de equipamentos com maior eficiência energética & 100 & 4 \\
\hline Instalação de interruptores para desligamento de equipamentos & 25 & 1 \\
\hline Presença de Procedimentos Operacionais Padronizados para manutenção de equipamentos & 75 & 3 \\
\hline Calibração de instrumentos/equipamentos de medição (termômetros) & 50 & 2 \\
\hline Manutenção preditiva, preventiva e corretiva de equipamentos e sistemas de aquecimento e refrigeração & 50 & 2 \\
\hline Registro de manutenção de equipamentos em geral & 50 & 2 \\
\hline Registro periódico dos procedimentos de limpeza e manutenção do sistema de climatização & 50 & 2 \\
\hline Iluminação & $\%$ & $\mathbf{n}$ \\
\hline Sistema de iluminação natural adequada & 50 & 2 \\
\hline Uso exclusivo de lâmpadas fluorescentes & 100 & 4 \\
\hline Rebaixamento de luminárias & 0 & 0 \\
\hline Instalação de sensores de presença & 25 & 1 \\
\hline Desligamento programado de sistemas de iluminação & 0 & 0 \\
\hline Ventilação & $\%$ & n \\
\hline Sistema de ventilação natural adequado & 25 & 1 \\
\hline Sistemas de ar condicionadas em áreas onde a ventilação natural não pode fornecer resultados satisfatórios & 50 & 2 \\
\hline Energia & $\%$ & $\mathbf{n}$ \\
\hline Instalação de medidores individuais de eletricidade & 25 & 1 \\
\hline Uso de painéis solares ou outras energias limpas para aquecimento de água & 25 & 1 \\
\hline Média da Tabela 1 & \multicolumn{2}{|c|}{$41,2 \%$} \\
\hline
\end{tabular}

Todas as UANs avaliadas utilizavam exclusivamente lâmpadas fluorescentes (Tabela 1), assim como observado em unidades hospitalares por Magrini \& Basso (2016). Porém, ainda eram deficitárias na implementação de ações sustentáveis simples, como o uso de luminárias rebaixadas e de sistemas de iluminação com desligamento programado por sensores de presença em locais onde há pouca atividade de produção ou de pessoal. Além disso, a maioria das unidades não dispunha de sistemas de iluminação e de ventilação naturais adequados ou utilizavam fontes alternativas de energia (painéis solares). Estas ações sustentáveis podem reduzir o consumo de energia elétrica nas UANs e, consequentemente, os impactos na fauna, flora, pesca, agricultura de subsistência e comercial decorrentes do alagamento de áreas para a formação dos lagos das hidroelétricas (Ezcurra et al., 2019). 


\subsection{Planejamento de cardápios, aquisição e armazenamento de insumos}

O cardápio é um dos elementos mais significativos nas práticas sustentáveis em UANs. Preparações saborosas e adequadas aos clientes reduzem a probabilidade de serem desperdiçadas e, por isso, é importante a elaboração de um cardápio que seja atrativo para o cliente, para evitar perdas e consequente aumento de geração de resíduos (Pimenta \& Gomes, 2012). Em todas as UANs hospitalares avaliadas, o cardápio era planejado por nutricionistas e, durante este processo, eles buscavam reduzir as sobras alimentares. Neste sentido, a presença e a atuação adequada do nutricionista no planejamento do cardápio são primordiais para a adoção de ações determinantes para a promoção da sustentabilidade no estabelecimento (Süßbauer et al., 2019).

Tabela 2. Práticas de sustentabilidade ambiental adotadas durante o planejamento de cardápio, aquisição e armazenamento de insumos em Unidades de Alimentação e Nutrição de hospitais em Governador Valadares-MG, agosto de 2018.

\begin{tabular}{lcc}
\hline Planejamento de cardápio & $\mathbf{\%}$ & $\mathbf{n}$ \\
\hline Cardápio planejado por nutricionista & 100 & 4 \\
\hline Cardápio planejado focando a redução de sobras & 100 & 4 \\
\hline Presença de fichas técnicas de preparação & 75 & 3 \\
\hline Uso de alimentos sazonais & 100 & 4 \\
\hline Média do item & $93,4 \%$ \\
\hline Aquisição de insumos & $\mathbf{\%}$ & $\mathbf{n}$ \\
\hline Uso de alimentos da agricultura familiar & 0 & 0 \\
\hline Aquisição de produtos orgânicos & 0 & 0 \\
\hline Aquisição de produtos em embalagens econômicas & 25 & 1 \\
\hline Média do item & $8,3 \%$ \\
\hline Armazenamento de insumos & $\mathbf{\%}$ & $\mathbf{n}$ \\
\hline Controle de temperatura & 50 & 2 \\
\hline Avaliação sensorial & 100 & 4 \\
\hline Média do item & $75,0 \%$
\end{tabular}

Verificou-se que $25 \%$ das UANs participantes não dispunham de ficha técnica de preparo (FTP) de alimentos (Tabela 2). As FTP são instrumentos que podem contribuir sobremaneira para diminuir as perdas de alimentos e a produção de resíduos que podem gerar impactos ambientais. Estas fichas padronizam as preparações quanto ao tipo e à quantidade de matérias-primas, e às técnicas de preparo, e, desta forma, subsidiam a aquisição de gêneros em quantidade adequada (Ferraz, 2014). Além disso, as FTPs podem abordar o tipo de calor empregado e sua intensidade, os utensílios, os equipamentos e a qualificação da mão de obra necessários, interferindo diretamente no resultado final do prato e na sustentabilidade ambiental, uma vez que faz menção às fontes de energia empregadas (Ferraz, 2014).

Outra importante ação ambientalmente sustentável adotada em todos os estabelecimentos foi a inclusão de alimentos sazonais no cardápio. O planejamento e a execução de cardápios sazonais, ou seja, que levem em conta a diversidade de alimentos regionais com regras sanitárias específicas, permitem diminuir os custos energéticos, de tempo, embalagem e transporte inerentes à importação de alimentos (Camillo et al., 2010).

Durante o processo de aquisição de insumos, verificou-se que apenas $8,3 \%$ das ações eram realizadas para a promoção da sustentabilidade ambiental (Tabela 2). Nenhuma das unidades adquiria alimentos orgânicos ou produzidos pela agricultura familiar. Estudos científicos sobre a utilização de alimentos orgânicos ou originados na agricultura familiar em UANs hospitalares não estão disponíveis. As agriculturas orgânica e familiar são reconhecidas como sistemas agroalimentares saudáveis e sustentáveis, ou seja, que reduzem ao mínimo o impacto ambiental sem comprometer a eficiência produtiva e sem esgotar os recursos naturais, e que contribuem para o incentivo à produção tradicional, com desenvolvimento social e econômico da região (Perramon et al., 2014).

Em todas as unidades, as matérias-primas eram analisadas sensorialmente durante o recebimento, porém, apenas em metade das UANs, a inspeção da temperatura dos insumos era realizada. A garantia da qualidade 
durante o recebimento das matérias-primas pode diminuir a perda de matérias-primas durante o armazenamento e a quantidade de resíduos gerados devido à baixa qualidade higienicossanitária e sensorial (Meybeck \& Redfern, 2016). Adicionalmente, estas medidas contribuem para minimizar a demanda por mais matéria-prima e os impactos gerados durante a sua cadeia produtiva, como, por exemplo, o consumo de "água virtual" necessária para a produção das matérias-primas ou a contaminação do ar pelos veículos movidos a combustíveis derivados do petróleo utilizados para transportá-las (Meybeck \& Redfern, 2016).

\subsection{Preparo, distribuição e consumo das refeições}

Na maioria das UANs, o descongelamento de carnes não era realizado em água corrente, minimizando assim o desperdício de água (Tabela 3). Apenas 50\% das unidades avaliadas realizavam o aproveitamento integral dos alimentos durante o preparo das refeições, percentual inferior ao observado por outros autores em UANs hospitalares (Magrini \& Basso, 2016). Destaca-se que a inclusão de cascas, folhas, talos e brotos, além de reduzir os resíduos gerados pela UAN, contribui para aumentar o valor nutricional das preparações, uma vez que muitas destas partes cotidianamente descartadas possuem valor nutricional superior ao das popularmente consumidas (Storck et al., 2013).

Apesar de as preparações serem avaliadas sensorialmente durante o preparo em todas as UANs, o controle de temperatura durante o preparo, a espera para distribuição e a distribuição de refeições ocorreram em 62,5\% das unidades. $\mathrm{O}$ controle de temperatura durante o preparo e a espera para a distribuição permitem o aproveitamento seguro das preparações não expostas para os comensais e contribuem para a aceitação das preparações expostas, reduzindo, assim, eventuais desperdícios de alimentos devido a inadequações microbiológica ou de temperatura (Gonçalves et al., 2018).

Tabela 3. Práticas de sustentabilidade ambiental adotadas durante o preparo, a distribuição e o consumo de refeições em Unidades de Alimentação e Nutrição de hospitais em Governador Valadares-MG, agosto de 2018.

\begin{tabular}{lcc}
\hline Preparo & $\mathbf{\%}$ & $\mathbf{n}$ \\
\hline Descongelamento em refrigerador, forno de conveç̧ão ou micro-ondas & 75 & 3 \\
\hline Aproveitamento integral dos alimentos & 50 & 2 \\
\hline Avaliação sensorial das preparações & 100 & 4 \\
\hline Controle de temperatura durante o preparo & 75 & 3 \\
\hline Média do item & $75,0 \%$ \\
\hline Distribuição e consumo & $\mathbf{\%}$ & $\mathbf{n}$ \\
\hline Controle de temperatura durante a espera e a distribuição de refeições & 50 & 2 \\
\hline Padronização da gramatura das porções & 100 & 4 \\
\hline Utilização de embalagens recicláveis para as refeições transportadas & 75 & 3 \\
\hline Controle de resto-ingestão por meio de cálculo de índice & 50 & 2 \\
\hline Manutenção de registro diário dos restos alimentares & 25 & 1 \\
\hline Controle de sobras limpas por meio de percentual de preparações & 25 \\
\hline Manutenção de registro diário de sobras das preparações & 50 \\
\hline Aproveitamento de sobras em pratos alternativos ou em refeições subsequentes & 2 \\
\hline Média do item & 75 \\
\hline
\end{tabular}

Em todas as unidades, as porções das preparações possuíam gramatura padronizada. A padronização adequada das porções considerando as necessidades energéticas do público-alvo diminui a oferta de alimentos em excesso ao cliente, reduzindo, assim, o desperdício de alimentos, bem como o consumo de energia e água durante a produção das refeições e de materiais descartáveis usualmente utilizados na distribuição de refeições em UANs hospitalares. 
O registro diário de índices e o cálculo de percentuais eram utilizados para controlar o resto alimentar e as sobras em menos de $50 \%$ dos estabelecimentos. A realização destes controles permite analisar, direta ou indiretamente, a adequação da quantidade preparada, do porcionamento e da aceitação das refeições, o que pode afetar o volume de resíduos produzidos pela UAN. O desperdício de alimentos é o maior desafio para a segurança alimentar global e uma importante alavanca para reduzir os impactos ambientais em Unidades de Alimentação e Nutrição, incluindo as hospitalares (Krishnan et al., 2020; Read et al., 2020). Desta forma, o controle de resto alimentar se torna fundamental para o planejamento e a avaliação de campanhas de educação alimentar e nutricional junto aos clientes, visando ao consumo consciente de alimentos e à redução do desperdício alimentar. Especialmente, em UANs hospitalares, é observado um percentual de resto-ingestão superior ao recomendado de, no máximo, 10\% (Nonino et al., 2012), devido à diversidade e às condições de saúde do seu público. Estudos demonstraram que o resto-ingestão nessas UANs variou de $20,80 \%$ a $28 \%$ do volume de refeições distribuídas a pacientes (Fonseca Novinski et al., 2017; Copatti et al., 2019).

Apesar do controle incipiente de temperatura durante a espera para a distribuição e a distribuição de refeições, as sobras limpas eram aproveitadas na maioria das unidades para o preparo de pratos alternativos ou em refeições subsequentes. Ressalta-se que, embora o aproveitamento das preparações reduza os impactos no meio ambiente, este deve ocorrer desde que garantida a qualidade higienicossanitária da refeição mediante um rigoroso controle do binômio tempo-temperatura.

\subsection{Higienização}

Todas as unidades possuíam Procedimentos Operacionais Padronizados (POPs) para higienização das instalações, dos equipamentos, móveis e utensílios, dos coletores de resíduos e da área de armazenamento (Tabela 4). Embora, durante o processo de higienização, todas as unidades utilizassem quantidade suficiente de detergente, uma unidade não realizava o enxágue controlado durante higienização, sendo este resultado semelhante ao observado em UANs hospitalares por Magrini e Basso (2016). Nenhuma unidade utilizava produtos biodegradáveis para a limpeza do local, os quais provocam menos impacto ao meio ambiente por serem degradados por microrganismos presentes na natureza (Gonçalves et al., 2015).

Tabela 4. Práticas de sustentabilidade ambiental adotadas durante a higienização em Unidades de Alimentação e Nutrição hospitalares de Governador Valadares-MG, agosto de 2018.

\begin{tabular}{lcc}
\hline Ações de higienização & $\%$ & $\mathbf{n}$ \\
\hline Higienização das instalações, dos equipamentos, dos móveis e utensílios & 100 & 4 \\
\hline Higienização de coletores de resíduos e da área de armazenamento & 100 & 4 \\
\hline Utilização de quantidade suficiente de detergente durante higienização & 100 & 4 \\
\hline Realização de enxágue controlado na higienização & 75 & 3 \\
\hline Aquisição de materiais de limpeza biodegradáveis & 0 & 0 \\
\hline Média do item & $93,8 \%$ \\
\hline
\end{tabular}

\subsection{Gestão de resíduos}

Embora importante para a promoção da sustentabilidade ambiental, a maioria das unidades não dispunha de POPs ou de um programa para a gestão de resíduos que abrangesse desde a coleta até a destinação final do resíduo (Tabela 5). A implementação do manifesto de resíduos em uma UAN é bastante apropriada uma vez que ele estabelece os procedimentos para a movimentação e destinação de resíduos sólidos e rejeitos, tornando obrigatória a emissão de Declaração de Movimentação de determinadas classes de resíduos sólidos e Certidão de Destinação, que contêm informações sobre o resíduo a ser encaminhado para a destinação, o gerador, o transportador e o destinador (Minas Gerais, 2019). 
Tabela 5. Práticas de sustentabilidade ambiental adotadas relacionadas à gestão de resíduos nas UANs hospitalares de Governador Valadares-MG, agosto de 2018.

\begin{tabular}{lcc}
\hline Aspectos Gerais & $\mathbf{\%}$ & $\mathbf{n}$ \\
\hline Procedimentos operacionais padronizados para o manejo de resíduos & 25 & 1 \\
\hline Programa de gestão integrada e sustentável dos resíduos gerados & 25 & 1 \\
\hline Coleta e armazenamento de resíduos & $\mathbf{\%}$ & $\mathbf{n}$ \\
\hline Presença de recipiente de lixo & 100 & 4 \\
\hline Localização adequada de recipiente de lixo & 100 & 4 \\
\hline Recolhimento frequente do lixo das áreas de produção & 100 & 4 \\
\hline Área de armazenamento para resíduos sólidos adequada & 75 & 3 \\
\hline Separação de resíduos recicláveis & 75 & 3 \\
\hline Plástico, vidro e papelão & 75 \\
\hline Isopor e tetra pak & 25 \\
\hline Resíduos pastosos e/ou aqueles de que escorrem líquido colocados em recipientes rígidos & 1 \\
\hline Retirada do lixo em horário e local diferente das matérias-primas & 75 \\
\hline Destinação de resíduos & $\mathbf{\%}$ & $\mathbf{n}$ \\
\hline Reciclagem de resíduos inorgânicos & 75 \\
\hline Reutilização de embalagens & 3 \\
\hline Reciclagem de resíduos sólidos orgânicos & 0 \\
\hline Tratamento de resíduos sólidos e líquidos orgânicos & 75 \\
\hline Comercialização/doação de resíduos de alimentos para empresas especializadas & 3 \\
\hline Registro de comercialização/doação de resíduos de alimentos & 50 \\
\hline Arquivamento de notas fiscais de serviços prestados pela empresa especializada na coleta de resíduos & 0 \\
\hline Acondicionamento de óleo de fritura em recipiente adequado, quando comercializado & 2 \\
\hline Registro da comercialização/doação de óleo de fritura & 50 \\
\hline Média do item & 0 \\
\hline
\end{tabular}

Todas as unidades dispunham de recipientes de lixo em quantidade adequada, recolhiam frequentemente o lixo das áreas de produção e separavam plástico, vidro e papel. Em estudos realizados em UAN hospitalares, verificou-se que mais de 50\% destes estabelecimentos separavam o lixo inorgânico (Magrini \& Basso, 2016), sendo o papelão/papel e o metal os itens mais separados (Santos, 2015). A separação seletiva dos resíduos é uma condição fundamental para o reaproveitamento dos resíduos inorgânicos, fazendo com que eles deixem de ser uma fonte de degradação para o meio ambiente ao sobrecarregar aterros/lixões (Lima Filho, 2016).

Embora o armazenamento dos resíduos sólidos fosse adequado na maioria dos estabelecimentos, em apenas $50 \%$ destes, os resíduos pastosos ou líquidos, incluindo óleo de fritura, eram adequadamente armazenados (Tabela 5). Estes resíduos, quando descartados inadequadamente, podem contaminar rios e lençóis freáticos, afetando a vida aquática e comprometendo a alimentação humana. Destaca-se que o óleo de fritura adequadamente armazenado deve ser encaminhamento para reciclagem, o que reduz o impacto no meio ambiente por evitar o seu descarte inapropriado e disposição inadequada em meio aquático (Goh et al., 2020).

A maioria das unidades (75\%) encaminhava os resíduos inorgânicos para reciclagem e nenhuma reutilizava embalagens. Além disso, nenhuma unidade mantinha notas fiscais de serviços prestados pela empresa especializada em coleta de resíduos, registro de comercialização de resíduos de alimentos ou de doação/comercialização de óleo para fritura. A gestão da produção de refeições deve ser realizada visando prioritariamente reduzir o volume de resíduos gerados durante este processo. Contudo, uma vez que ao menos quantidades mínimas de resíduos serão geradas, torna-se necessário adotar soluções para minimizar o seu impacto ao meio ambiente, tais como a coleta seletiva e a reciclagem (Dhir et al., 2020) 


\section{Conclusão}

Apesar de as Unidades de Alimentação e Nutrição de Hospitais de Governador Valadares realizarem algum tipo de ação para promover a sustentabilidade ambiental, foram identificadas também ações que não são sustentáveis. Ressalta-se que práticas simples, tais como a presença de sistema de iluminação adequado, a realização do controle de temperatura dos alimentos, do aproveitamento integral de alimentos e do controle de resto, não eram desempenhadas em algumas unidades.

As ações mais prevalentes ocorriam durante o planejamento de cardápio, o preparo e a distribuição de refeições. Ainda se mostra necessário serem intensificadas ações relacionadas a estrutura física, aquisição e recepção de insumos, e gestão de resíduos. Destaca-se ainda a necessidade de o tema sustentabilidade ambiental ser abordado de maneira mais frequente na formação do nutricionista e dos colaboradores e clientes da UAN.

\section{Referências}

Araújo, E. L. M., \& Carvalho, A. C. M. S. (2015). Sustentabilidade e geração de resíduos em uma unidade de alimentação e nutrição da cidade de Goiânia-GO. DEMETRA: Alimentação, Nutrição \& Saúde, 10(4), 775-796. http://dx.doi.org/10.12957/demetra.2015.17035

Block, B. (2019). Recuperado em 19 de abril de 2021, de http://www.worldwatch.org/node/5661

Camillo, A., Kim, W. G., Moreo, P. J., \& Ryan, B. (2010). A model of historical development and future trends of Italian cuisine in America. International Journal of Hospitality Management, 29(4), 549-558.http://dx.doi.org/10.1016/j.ijhm.2009.09.002

Carino, S., Porter, J., Malekpour, S., \& Collins, J. (2020). Environmental sustainability of hospital foodservices across the food supply chain: A systematic review. Journal of the Academy of Nutrition and Dietetics, 120(5), 825-873. PMid:32093919. http://dx.doi.org/10.1016/j.jand.2020.01.001

Colares, L. G. T., Figueiredo, V. O., Ferreira, A. A., \& Oliveira, A. G. M. (2018). Lista de verificação de boas práticas ambientais para serviços de alimentação: Elaboração, validação de conteúdo e confiabilidade interavaliadores. Brazilian Journal of Food Technology, 21, e2017066. https://doi.org/https://doi.org/10.1590/1981-6723.

Copatti, L. C., Bruger, V. B., Baratto, I., \& Brufati, A. (2019). Avaliação de resto de ingesta em uma unidade de alimentação e nutrição hospitalar na cidade de Pato Branco-PR. Revista Brasileira de Obesidade, Nutrição e Emagrecimento, 12(75), 976-983.

Dhir, A., Talwar, S., Kaur, P., \& Malibari, A. (2020). Food waste in hospitality and food services: A systematic literature review and framework development approach. Journal of Cleaner Production, 270, 122861.

http://dx.doi.org/10.1016/j.jclepro.2020.122861

Ezcurra, E., Barrios, E., Ezcurra, P., Ezcurra, A., Vanderplank, S., Vidal, O., Villanueva-Almanza, L., \& Aburto-Oropeza, O. (2019). A natural experiment reveals the impact of hydroelectric dams on the estuaries of tropical rivers. Science Advances 5(3), eaau9875. PMid:30891500. http://dx.doi.org/10.1126/sciadv.aau9875

Ferraz, R. R. N. (2014). Redução de custos e melhoria dos padrões de gestão com a utilização de fichas técnicas de preparação para padronização da montagem de refeições em unidades hospitalares. Revista dos Mestrados Profissionais, 2(2), 1-13.

Fonseca Novinski, A. P., Araújo, G. C., \& Baratto, I. (2017). Resto ingesta em uma unidade de alimentação e nutrição hospitalar na cidade de Pato Branco-PR. Revista Brasileira de Obesidade. Nutrição e Emagrecimento, 11(66), 451-458.

Goh, B. H. H., Chong, C. T., Ge, Y., Ong, H. C., Ng, J. H., Tian, B., \& Józsa, V. (2020). Progress in utilisation of waste cooking oil for sustainable biodiesel and biojet fuel production. Energy Conversion and Management, 223, 113296.

Gonçalves, E., Bozzi, J. T., Mazolini, L. T., Oliveira, R. C., Zanin, C. I. C. B., \& Lima, A. A. (2015). Tensoativos biodegradáveis. Gestão em Foco, 7, 252-258.

Gonçalves, L. C., Silveira, C. D. S., Pereira, M. C., \& Helbig, E. (2018). Sustentabilidade ambiental em restaurantes comerciais da zona central de Pelotas-RS. Revista Gestão \& Sustentabilidade Ambiental, 7(2), 525-539. http://dx.doi.org/10.19177/rgsa.v7e22018525-539

González-García, S., González-García, R., González Vázquez, L., Moreira, M. T., \& Leis, R. (2020). Tracking the environmental footprints of institutional restaurant service in nursery schools. The Science of the Total Environment, 728, 138939. PMid:32361112. http://dx.doi.org/10.1016/j.scitotenv.2020.138939

Hatjiathanassiadou, M., Souza, S. R. G. D., Nogueira, J. P., Oliveira, L. D. M., Strasburg, V. J., Rolim, P. M., \& Seabra, L. M. A. J. (2019). Environmental impacts of university restaurant menus: A case study in Brazil. Sustainability, 11(19), 5157. http://dx.doi.org/10.3390/su11195157

Krishnan, R., Agarwal, R., Bajada, C., \& Arshinder, K. (2020). Redesigning a food supply chain for environmental sustainability: An analysis of resource use and recovery. Journal of Cleaner Production, 242, 118374. http://dx.doi.org/10.1016/j.jclepro.2019.118374

Lima Filho, G. D. (2016). Separação seletiva e reciclagem como instrumento para conservação ambiental: Estudo de caso no lixão do município de Sumé-Paraíba. Revista Científica Semana Acadêmica, 1, 1-15. 
Llach, J., Perramon, J., del Mar Alonso-Almeida, M., \& Bagur-Femenías, L. (2013). Joint impact of quality and environmental practices on firm performance in small service businesses: An empirical study of restaurants. Journal of Cleaner Production, 44 , 96-104. http://dx.doi.org/10.1016/j.jclepro.2012.10.046

Magrini, L. N., \& Basso, C. (2016). Práticas sustentáveis em serviço de alimentação hospitalar. Disciplinarum Scientia Saúde, 17(2), 257-265.

Meybeck, A., \& Redfern, S. (2016). Sustainable value chains for sustainable food systems: A workshop of the FAO/UNEP Programme on Sustainable Food Systems. Rome: FAO.

Minas Gerais. Conselho de Política Ambiental - COPAM. (2019, março 9). Deliberação Normativa n² 232, de 27 de dezembro de 2019. Institui o Sistema Estadual de Manifesto de Transporte de Resíduos e estabelece procedimentos para o controle de movimentação e destinação de resíduos sólidos e rejeitos no estado de Minas Gerais e dá outras providências. Diário do Executivo - "Minas Gerais", Belo Horizonte.

Moskwa, E., Higgins-Desbiolles, F., \& Gifford, S. (2015). Sustainability through food and conversation: The role of an entrepreneurial restaurateur in fostering engagement with sustainable development issues. Journal of Sustainable Tourism, 23(1), 126-145. http://dx.doi.org/10.1080/09669582.2014.940046

Nonino, C. B., Tanaka, Y. Y., \& Marchini, J. S. (2012). Controle do desperdício e manejo de resíduos. In M. C. H. Vieira \& C. C. Japur (Eds.), Gestão de qualidade na produção de refeições (1. ed., Cap. 23, pp. 258-265). Rio de Janeiro: Guanabara Koogan. Payne-Palacio, J., \& Theis, M. (2015). Foodservice management. London: Pearson Education.

Perramon, J., del Mar Alonso-Almeida, M., Llach, J., \& Bagur-Femenías, L. (2014). Green practices in restaurants: Impact on firm performance. Operations Management Research, 7(1-2), 2-12. http://dx.doi.org/10.1007/s12063-014-0084-y

Pimenta, A. S., \& Gomes, A. M. (2012). Alimentação saudável sustentabilidade e redução do desperdício alimentar nos hospitais. Hotelaria \& Saúde, 2, 12-14.

Read, Q. D., Brown, S., Cuéllar, A. D., Finn, S. M., Gephart, J. A., Marston, L. T., Meyer, E., Weitz, K. A., \& Muth, M. K. (2020). Assessing the environmental impacts of halving food loss and waste along the food supply chain. The Science of the Total Environment, 712, 136255. PMid:32050400. http://dx.doi.org/10.1016/j.scitotenv.2019.136255

Santos, A. V. (2015). Avaliação da geração de resíduos de embalagens em um serviço de nutrição e dietética hospitalar público (Trabalho de conclusão de curso). Universidade Federal do Rio Grande do Sul, Porto Alegre.

Sgroi, M., Vagliasindi, F. G., \& Roccaro, P. (2018). Conceitos de viabilidade, sustentabilidade e economia circular no reúso de água. Opinião Atual em Ciência e Saúde Ambiental, 2, 20-25.

Silva, S. (2017). Restaurantes: Estudo sobre o aproveitamento da matéria prima e impactos das sobras no meio ambiente (Dissertação de mestrado). Universidade de Taubaté, Taubaté.

Storck, C. R., Nunes, G. L., Oliveira, B. B., \& Basso, C. (2013). Folhas, talos, cascas e sementes de vegetais: Composição nutricional, aproveitamento na alimentação e análise sensorial de preparações. Ciência Rural, 43(3), 537-543. http://dx.doi.org/10.1590/S0103-84782013000300027

Süßbauer, E., Maas-Deipenbrock, R. M., Friedrich, S., Kreß-Ludwig, M., Langen, N., \& Muster, V. (2019). Employee roles in sustainability transformation processes: A move away from expertise and towards experience-driven sustainability management. GAIA-Ecological Perspectives for Science and Society, 28(1), 210-217. http://dx.doi.org/10.14512/gaia.28.S1.7

Viana, R. M., \& Ferreira, L. C. (2017). Avaliação do desperdício de alimentos em uma unidade de alimentação e nutrição na cidade de Januária-MG. Higiene Alimentar, 31(266-267), 22-26.

Yucedag, C., Kaya, L. G., \& Cetin, M. (2018). Identifying and assessing environmental awareness of hotel and restaurant employees' attitudes in the Amasra District of Bartin. Environmental Monitoring and Assessment, 190(2), 60. PMid:29305729. http://dx.doi.org/10.1007/s10661-017-6456-7

Zotesso, J. P., Cossich, E. S., Colares, L., \& Tavares, C. R. G. (2016). Avaliação do desperdício de alimentos e sua relação com a geração de resíduos sólidos em um restaurante universitário. Engevista, 18(2), 294-308.

http://dx.doi.org/10.22409/engevista.v18i2.730

Financiamento: Nenhum.

Received: Apr. 21, 2020; Accepted: June 02, 2021

Section Editor: Silvia P. M. Germer 